\title{
Construção e validação de um guia da rede SUS em um município da Baixada
}

\author{
Maranhense \\ Construction and validation of a SUS network guide in a municipality of Baixada Maranhense \\ Construcción y validación de una guía de red SUS en un município de Baixada Maranhense
}

Recebido: 28/12/2021 | Revisado: 03/01/2022 | Aceito: 07/01/2022 | Publicado: 11/01/2022

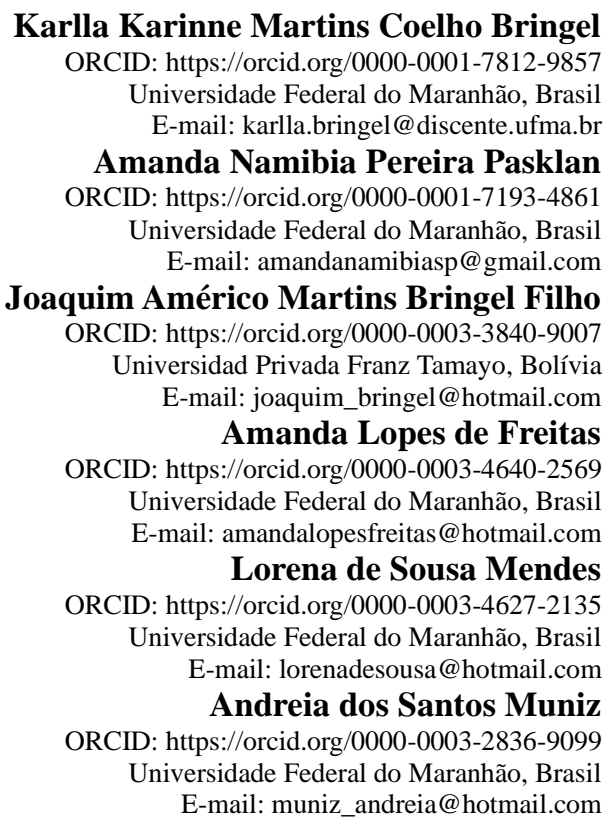

\begin{abstract}
Resumo
Objetivo: Construir e validar um Guia da Rede SUS em Pinheiro-MA. Metodologia: Trata-se de uma pesquisa metodológica descritiva, de natureza qualitativa e documental, que foi desenvolvida nas seguintes etapas: (1) mapeamento das unidades de atendimento no município, (2) diagnóstico situacional dos serviços de assistência à saúde, (3) construção do Guia da Rede SUS em Pinheiro-MA e (4) validação local desse instrumento. Após a construção do Guia a partir das informações disponibilizadas pelos coordenadores das 44 unidades de atendimento disponíveis, partiu-se para a etapa de validação que, por sua vez, contou com a participação de 04 gestores, 05 profissionais da rede, 05 acadêmicos da área da saúde e 05 usuários. Todos os envolvidos precisaram atender a critérios de inclusão para que pudessem ser considerados juízes nesse processo. Os itens avaliados foram: "Linguagem", "Acessibilidade", "Organização" e "Relevância". Para fins de Validação, a porcentagem de concordância mínima estabelecida entre os juízes-especialistas precisou garantir um Coeficiente de Validade de Conteúdo (CVC) superior a 80\%. Resultados: Atingiu-se um CVC de 93,42\%, ou seja, acima do valor mínimo tolerado. Conclusão: O Guia construído atendeu a todos os aspectos, sendo considerado satisfatório para sua validação.
\end{abstract}

Palavras-chave: Sistema Único de Saúde; Assistência integral à saúde; Tecnologia da informação; Guias informativos; Estudo de validação.

\begin{abstract}
Objective: To build and validate a Guide of the SUS Network in Pinheiro-MA. Methodology: This is a descriptive methodological research, qualitative and documentary in nature, which was developed in the following stages: (1) mapping of care units in the municipality, (2) situational diagnosis of health care services, (3) construction of the SUS Network Guide in Pinheiro-MA and (4) local validation of this instrument. After the construction of the Guide from the information provided by the coordinators of the 44 service units available, we went to the validation stage, which, in turn, had the participations of 04 managers, 05 network professionals, 05 health students and 05 users. All those involved had to meet inclusion criteria so that they could be considered judges in this process. The items evaluated were: "Language", "Accessibility", "Organization" and "Relevance". For validation purposes, the percentage of minimum agreement established among the expert judges had to ensure a Content Validity Coefficient (cvc) greater
\end{abstract}


than $80 \%$. Results: A CVC of $93,42 \%$ was reached, that is, above the minimum tolerated value. Conclusion: The constructed Guide met all aspects been considered satisfactory for its validation.

Keywords: Unified Health System; Comprehensive health care; Information technology; Informative guides; Validation study.

\section{Resumen}

Objetivo: Construir y validar una Guía de Red SUS en Pinheiro-MA. Metodología: Se trata de una investigación metodológica descriptiva, de carácter cualitativo y documental, que se desarrolló en las siguientes etapas: (1) mapeo de las unidades de salud del municipio, (2) diagnóstico situacional de los servicios de salud, (3) construcción de la Guía de la Red SUS en Pinheiro-MA y (4) validación local de este instrumento. Luego de la construcción de la Guía en base a la información brindada por los coordinadores de las 44 unidades de servicio disponibles, se dio la etapa de validación, la cual, a su vez, contó con la participación de 04 gerentes, 05 profesionales de la red, 05 académicos del área. y 05 usuarios. Todos los involucrados debían cumplir con los criterios de inclusión para poder ser considerados jueces en este proceso. Los ítems evaluados fueron: "Idioma", "Accesibilidad", "Organización" y "Relevancia". A efectos de Validación, el porcentaje mínimo de acuerdo establecido entre los jueces expertos necesario para garantizar un Coeficiente de Validez de Contenido (CVC) superior al 80\%. Resultados: Se alcanzó un CVC de 93,42\%, es decir, por encima del valor mínimo tolerado. Conclusión: La Guía elaborada cumplió con todos los aspectos, siendo así considerado satisfactorio para su validación.

Palabras clave: Sistema Único de Salud; Atención integral de salud; Tecnología de la información; Guías informativas; Estudio de validación.

\section{Introdução}

O Sistema Único de Saúde (SUS) foi, nos últimos anos, a reforma setorial da saúde mais importante no cenário mundial. Sua constituição prevê a implementação de uma rede regionalizada, descentralizada e com ampla participação popular, além de propor como princípios éticos e doutrinários a universalidade, a integralidade e a equidade no acesso à saúde.

Trata-se de um Sistema composto de três níveis de Atenção à Saúde que devem se intercomunicar em rede: básica, média e alta complexidade. Está estruturado de forma hierarquizada, com comando único nas respectivas esferas de gestão federal, representada pelo Ministério da Saúde; estadual, organizada nas Secretarias Estaduais de Saúde, e municipal, representada pelas Secretarias Municipais de Saúde (Ministério da Saúde, 2019).

No que diz respeito ao seu princípio descentralizador, por exemplo, o SUS formaliza o reconhecimento de que o município é o principal responsável pelo completo estado de bem-estar físico, mental e social da população. Ou seja, estabelecer uma parceria sólida com os municípios é a grande oportunidade de conscientizar os cidadãos de que investir em promoção da saúde é melhor do que nutrir o círculo vicioso de agravamento de doenças, que geram gastos cada vez mais elevados, com a consequente redução da qualidade de vida. Nesse contexto, os gestores do SUS vêm se empenhando continuamente em planejar, monitorar e avaliar as inúmeras ações e serviços em saúde. Tais esforços têm contribuído, certamente, para notórios avanços registrados pelo SUS desde sua criação. Contudo, é válido reconhecer que os desafios atuais exigem novos posicionamentos, capazes de favorecer a aplicação de toda a sua potencialidade, corroborando de forma plena e efetiva para a consolidação deste Sistema (Luz, et al., 2020). É impreterível, portanto, que a gestão invista em políticas sociais e econômicas coerentes com a realidade local, levando à redução das desigualdades sociais e regionais em nosso País, assegurando a cidadania e fortalecendo a democracia.

Ademais, é também responsabilidade dos municípios garantirem meios para que ocorra a participação dos cidadãos no contexto da assistência à saúde, colocando-os na condição de participantes ativos capazes de envolverem-se em debates, formulações e fiscalização das políticas desenvolvidas pela saúde pública. Além disso, a inserção de novos sujeitos sociais nos processos de gestão do SUS permite a ampliação do acesso à informação e às orientações de cuidado, além de assegurar legitimidade e transparência ao Sistema Único de Saúde. Por extensão, o entendimento integral sobre a oferta da rede de saúde facilita também a interdisciplinaridade dos profissionais no enfrentamento de cada situação identificada, objetivando propor soluções conjuntas e intervir de maneira adequada conforme cada nível de atenção (Gil, 2016). 
Nessa perspectiva, visando ainda garantir a integralidade e o acesso universal aos serviços de saúde, é papel da gestão municipal promover a integração entre todos os pontos de atenção à saúde no município (Silva, 2018). Para que isso ocorra, é fundamental que cada serviço de saúde conheça o território sob sua responsabilidade e os compromissos assumidos com a gestão do SUS, de modo a pensar estratégias direcionadas às necessidades locais, bem como intervir nas principais demandas. Assim sendo, parte-se do princípio de que todos os usuários devam ser assistidos por uma rede de Atenção Integral que garanta um atendimento multidisciplinar, focado no ser humano como um todo e baseado na interação entre tratamento, prevenção e promoção da saúde pública (Alves, 2017).

Essa proposta de cuidado, contudo, esbarra no confronto com a falta de articulação e organização da rede, além da carência de divulgações sobre o funcionamento dos serviços e a própria cultura da institucionalização, que tende a centrar o trabalho dentro das unidades (Kemper et al., 2015). Na prática, ainda que o SUS tenha avançado bastante na concretização de alguns de seus princípios, a área da saúde é ainda alvo na disputa de interesses econômicos, políticos e de diferentes projetos com ação de múltiplos atores sociais, tanto nos espaços de gestão do Estado como nos de participação e controle social. São disputas de poder que requerem decisões de política pública que certamente ferem interesses (Drumond \& Neto, 2021), limitando a execução daquilo que é preconizado, bem como a efetivação dos preceitos e implementação de políticas de saúde baseadas nos princípios do SUS (Guarnieri, 2017).

Diante dessa realidade, faz-se necessário pensar em estratégias eficazes que viabilizem uma assistência à saúde mais acessível, equitativa, integrativa e que satisfaça melhor as expectativas dos cidadãos. Nesse contexto, como importante alternativa a serviço da gestão, tem-se a criação de instrumentos que mapeiem a rede de saúde, além de protocolos de organização dos serviços. Essas ferramentas abrangem os fluxos administrativos contidos na proposta dos serviços em rede, estabelecendo as interfaces entre as diversas unidades, entre os níveis de atenção e entre outras instituições sociais (Feliciello, 2021). Sendo assim, as ferramentas de normalização dos processos de trabalho em saúde assumem funções tanto de caráter gerencial, quanto educacional e comunicacional. A função gerencial, por exemplo, decorre da necessidade de controlar a lei da variabilidade clínica nos serviços de saúde. Por extensão, a função educacional determina que os instrumentos de normalização devam ser transformados em produtos de educação para profissionais e usuários do sistema de saúde. No que diz respeito à função de comunicação, por outro lado, a intenção é mudar o comportamento dos profissionais de saúde e dos usuários em relação à condição situacional, aumentando a adesão e a participação da comunidade no contexto da saúde pública.

Nessa perspectiva, as tecnologias de informação e comunicação são ferramentas que apresentam grande relevância na promoção de saúde. A sua utilização proporciona autonomia, autoestima e socialização de conhecimentos, tendo como resultado a mudança de comportamentos de saúde. Conforme os autores, os profissionais de saúde capacitados devem estar sempre em atualização, verificando as potencialidades das tecnologias, utilizando-as como estratégia para educação, disponibilização de informação e construção de conhecimento, além de contribuir com o desenvolvimento de um pensamento mais crítico por parte da comunidade. $\mathrm{O}$ fato é que a tecnologia em saúde mediante ações virtuais pode garantir maior abrangência e acesso à saúde, a educação e à prevenção. Dessa forma, é possível auxiliar no cumprimento dos princípios do Sistema Único de Saúde (SUS), reduzindo o tempo de espera, fornecendo atendimento integral, efetivo, com maior interação da equipe, logo, proporciona o atendimento por especialistas, um alcance maior de pessoas, diagnósticos mais precisos, além de promover o autocuidado e o compartilhamento de informações fundamentais para saúde (Thomas \& Fontana, 2020).

Nesse contexto, partindo do princípio de que o estudo em questão visa proporcionar a validação de um Guia da Rede SUS em um importante município da Baixada Maranhense, valem ressaltar os aspectos relacionados à descentralização das ações de saúde, seu caráter participativo e a integralidade da assistência. Em síntese, o objetivo com a construção de um instrumento editorial como esse é, portanto, organizar sistematicamente os serviços, garantir a otimização do trabalho, mantendo ou melhorando sua qualidade e fazendo cumprir com as funções para as quais foram criadas. 


\section{Metodologia}

Trata-se de uma pesquisa metodológica descritiva, de natureza qualitativa e documental, que foi desenvolvida nas seguintes etapas: mapeamento das unidades de atendimento; diagnóstico situacional dos serviços de assistência à saúde; construção do Guia da Rede SUS em Pinheiro-MA e validação local desse instrumento.

O estudo descritivo pretende descrever com exatidão os fatos e fenômenos de determinada realidade. Os estudos qualitativos, por sua vez, se caracterizam como aqueles que buscam compreender um fenômeno em seu ambiente natural, onde esses ocorrem e do qual faz parte. Para tanto o investigador é o instrumento principal por captar as informações, interessandose mais pelo processo do que pelo produto. As informações ou dados coletados podem ser obtidos e analisado de várias maneiras dependendo do objetivo que se deseja atingir. Num estudo qualitativo a busca por dados na investigação leva o pesquisador a percorrer caminhos diversos, isto é, utiliza uma variedade de procedimentos e instrumentos de constituição e análise de dados. Já a pesquisa documental propõe-se a produzir novos conhecimentos, criar novas formas de compreender os fenômenos e dar a conhecer a forma como estes têm sido desenvolvidos (Kripka et al., 2015).

A escolha dessa metodologia deu-se por entender que este é o caminho que melhor se encaixa ao objeto da pesquisa, além de englobar perfeitamente o objetivo e a problemática apresentada. Sendo assim, pesquisar utilizando essa abordagem significa procurar conhecer a realidade de um determinado ambiente partindo do seu contexto mais amplo, para então entender seus aspectos específicos. Nesse estudo, o local escolhido foi o município do Pinheiro-Maranhão, localizado na microrregião da Baixada Maranhense e caracterizado por uma população com múltiplas carências.

À priori, diante da aprovação pelo Comitê de Ética e Pesquisa da Universidade Federal do Maranhão, de acordo com as Resoluções 466/2012 e 580/2018, em Junho de 2021 foi realizado, com o auxílio da gestão municipal de saúde, o mapeamento dos serviços de saúde ofertados, além da sua organização didática nos seguintes setores: Atenção Primária à Saúde; Serviços Especializados; Atenção Hospitalar de Referência; Urgência e Emergência; Programas; Serviços de Assistência; Serviços Laboratoriais e Vigilância em Saúde. Em seguida, entre os meses de Julho e Agosto do mesmo ano, por meio de roteiros-guia também disponibilizados pela gestão municipal de saúde, foi realizada a etapa de coleta das informações referentes ao funcionamento das 44 unidades de atendimento em Pinheiro-MA. As entrevistas, em sua maioria, acorreram presencialmente no próprio local de funcionamento do serviço ou, quando solicitado, via Google Forms, conforme disponibilidade do entrevistado e do entrevistador. Ao fazer uso da entrevista como técnica na coleta de dados o investigador estabelece uma relação de interação com o informante, tornando esse cenário mais favorável para que as informações sejam as mais fiéis possíveis (Cruz \& Medeiros, 2021). O objetivo, nessa etapa de diagnóstico situacional dos serviços, foi compreender o funcionamento geral de cada uma das unidades e organizar as informações em tópicos como: dias e horários de funcionamento, informações consideradas importantes para marcação do atendimento; telefone para contato; endereço da unidade, nome do responsável, além de orientações consideradas essenciais para a melhor compreensão do serviço prestado. Além disso, foram realizados registros fotográficos das fachadas de todas as unidades frequentadas pelo pesquisador, exceto aquelas cuja entrevista não foi realizada presencialmente.

Posteriormente, ainda no mês de Agosto, diante de todas as informações devidamente coletadas e organizadas nos setores já descritos partiu-se para a etapa de construção efetiva do Guia. Para isso, utilizou-se como ferramenta o aplicativo Microsoft Power Point 2010, onde foi possível realizar a elaboração textual a partir do conteúdo, seguida da formatação e diagramação. A escolha pela organização dos setores em diferentes cores pretendeu organizar visualmente a leitura e tornar este instrumento ainda mais acessível para todos os públicos.

Após finalizada essa fase de construção do Guia, em Setembro de 2021 iniciou-se a etapa de validação local desse instrumento. Com essa finalidade, além do Guia em PDF, foram disponibilizados formulários com o Termo de Consentimento Livre e Esclarecido (TCLE), via Whatsapp, a vinte “juízes” previamente selecionados, são eles: 05 gestores em atuação no 
cargo por pelo menos três anos; 05 profissionais da rede em exercício de suas atividades por pelo menos três anos, dentre eles 01 Médico, 01 Enfermeiro, 01 Cirurgião Dentista, 01 Técnico de enfermagem e 01 Agente Comunitário de Saúde; 05 acadêmicos da área da saúde em fase de conclusão de curso na Universidade Federal do Maranhão-campus Pinheiro e 05 usuários, dentre eles líderes civis, que residem no município por pelo menos 03 anos e que tenham frequentado ao menos dois serviços públicos de saúde ofertados no município. A escolha dos públicos-alvo levou em consideração que o instrumento criado, uma vez disponibilizado, beneficiará igualmente a todos os seguimentos sociais envolvidos, sem quaisquer preferências. A intenção, pois, era que estivessem envolvidos nesse processo de validação pessoas com certo engajamento no contexto da Saúde Pública local, isto é, indivíduos capazes de opinar sobre um instrumento que retrata o funcionamento da Rede SUS no Município.

Quanto ao formulário de validação, foram levantados os seguintes aspectos: 1- identificação do entrevistado: no caso do gestor foi questionado o cargo de gestão e o tempo de trabalho; entre os profissionais da saúde questionada a formação acadêmica, o tempo de trabalho e os serviços em atuação no momento; entre os acadêmicos da área da saúde foi perguntado o curso, o período e os campos de prática frequentados e, por fim, entre os usuários, a idade, a atuação, o tempo de residência no município, além dos serviços de saúde frequentados entre o ano de 2020 e 2021; 2- avaliação da Linguagem em: "muito clara", "parcialmente clara" ou "confusa"; 3 - avaliação da Acessibilidade em: "fácil acesso às informações" ou "difícil acesso às informações"; 4 - avaliação da Organização em: "bem organizado", "parcialmente organizado" ou "desorganizado" e 5 avaliação da Relevância em: "relevante" ou "irrelevante". Além disso, ao final de cada questionário foi garantida a possibilidade de sugerir alterações ao Guia construído, as quais foram levadas em consideração na construção da versão final desse instrumento.

Finalizada essa etapa de validação, as respostas captadas receberam tratamento descritivo, foram avaliadas individualmente e organizadas na forma de tabelas, para melhor análise dos resultados. Em sequência, os dados foram disponibilizados em planilha Excel 2010 para criação de um gráfico resumo, a fim de facilitar ainda mais a interpretação das respostas obtidas. Para análise dos dados, por sua vez, utilizam-se os recursos da estatística aplicando o Índice de Validade de Conteúdo (IVC) que mede a proporção ou porcentagem de concordância entre os aspectos considerados pelo autor como fundamentais para a validação do instrumento. Sendo assim, para cada item do formulário o IVC foi calculado dividindo o número de respostas consideradas satisfatórias pelo total de respostas obtidas, como descrito na fórmula abaixo:

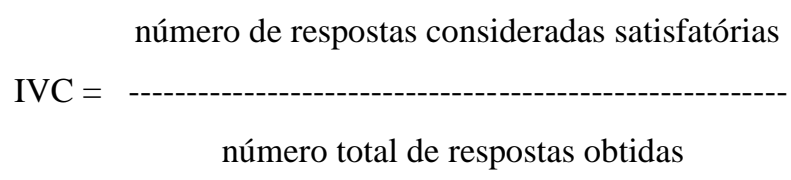

Para o item "Linguagem", por exemplo, foi considerado satisfatório pelo autor a opção "Muito clara"; para o item "Acessibilidade" a opção "Fácil acesso às informações"; para o item "Organização" a opção "Bem organizado" e para o item "Relevância" a opção "Muito relevante". A concordância do instrumento como um todo foi definida a partir da soma dos IVC de cada item e sua divisão pelo total geral de itens que compõem o instrumento, utilizando-se a seguinte fórmula:

soma de todos os IVC

$\%$ concordância $=$

total de itens. 
A avaliação do consenso entre os juízes, para fins de validação do Guia em questão, visa alcançar um Coeficiente de Validade de Conteúdo (CVC) com valor mínimo de 0,80 (80\%). Tal método, além de ser utilizado com frequência em pesquisas da área da saúde, objetiva a mensuração da porcentagem de concordância mínima estabelecida entre os juízesespecialistas.

\section{Resultados e Discussão}

O Guia, em sua versão final, foi estruturado da seguinte forma: I - Capa: com especificação do título, do ano de construção do Guia e dos públicos alvos; II - Ficha catalográfica; III - Corpo editorial: com nome, formação e e-mail do autor, além do nome e da descrição referente à formação da orientadora; IV - Apresentação: tópico de detalhamento sobre o SUS e sobre as Redes de Atenção à Saúde, deixado explícito os objetivos do instrumento; V - Agradecimento: espaço destinado aos agradecimentos do autor; VI - Sumário: lista constando os números das páginas onde estão registrados cada item do instrumento; VII - Corpo do Guia: descrição de cada serviço organizado em Atenção Primária à Saúde, Serviços Especializados, Atenção Hospitalar de Referência, Urgência e Emergência, Programas, Serviços de Assistência, Serviços Laboratoriais e Vigilância em Saúde. Inicialmente, para cada um desses tópicos são feitos esclarecimentos gerais seguidos de detalhamentos sobre cada uma das unidades. Além de informações textuais organizadas em tópicos, na maioria dos serviços foram adicionadas fotos da fachada, de modo a facilitar a identificação das unidades de atendimento. Por fim, na última página do Guia foi disponibilizado um QR Code (Figura 1) que viabiliza o acesso virtual ao Guia da Rede SUS em Pinheiro-MA, garantindo maior praticidade e facilidade no acesso às informações.

Figura1. QR Code do Guia da Rede SUS em Pinheiro-MA.

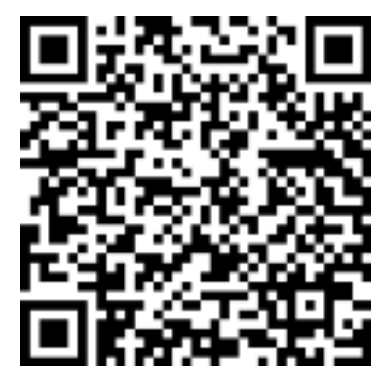

Fonte: Autores (2021).

A escolha por essa estruturação teve o intuito de facilitar a compreensão e organização do conteúdo, de forma a torná-la mais interativa, para que o leitor sinta-se motivado a seguir com a leitura, além de deixá-lo mais propenso a adotar novos comportamentos e facilitar seu acesso à saúde. Quanto à confecção de materiais educativos, a literatura reforça que devem ser atrativos, objetivos, não podendo ser extensos, mas capazes de dar uma orientação significativa sobre o tema a que se propõem, além de atender às necessidades específicas de uma determinada situação de saúde e ao público a que o material é destinado. Ademais, como forma de garantir a qualidade do material, é fundamento a realização da validação ou qualificação por especialistas no assunto. Tal análise tem o intuito de estabelecer a compreensão dos itens e a pertinência dos mesmos ao atributo que se propõe validar, ajuizando se os itens estão se referindo ou não ao objetivo em questão, além de analisar as possíveis sugestões que sejam apresentadas, visando à melhoria do material (Silva et al., 2021). Ainda, cabe destacar que, ao elaborar uma tecnologia do tipo digital é importante atentar-se para aspectos como organização e visualização, além de observar a quantidade de informação contida em cada tela, tamanho e tipo de fonte, escolha das cores e contrastes entre elas, bem como outros aspectos que culminem na fácil apresentação dos conteúdos (Campos et al., 2021). 
A proposta de construção deste instrumento representa o esboço de uma rede que se encontra em construção; alinhavada, pois, em fase de expansão, criação e organização dos serviços. Durante a sua elaboração, destacaram-se os seguintes fatores limitantes: a incompatibilidade de horários entre os envolvidos na entrevista; a distância física entre as unidades de atendimento no município, demandando ainda mais tempo e recursos financeiros para custeio dos deslocamentos; além do desafio de estruturar o Guia com o compromisso de adequar a linguagem textual e visual aos diferentes públicos, bem como garantir clareza e organização, selecionar bem as informações e buscar formas de viabilizar o acesso. Além disso, por se tratar de um município com uma população estimada de 84160 pessoas e que, portanto, conta com uma extensa lista de unidades de atendimento e serviços, foi inquestionavelmente desafiador estabelecer contato com os coordenadores de todas as 44 unidades de atendimento em atividade. Essa realidade levou a um retardo na construção do instrumento, com repercussões relacionadas à possibilidade de ampla divulgação do Guia. Sendo assim, tem-se como fator limitante o entendimento de que as informações coletadas nos meses de Julho e Agosto muito provavelmente não corresponderiam às informações de meses posteriores devido à dinâmica dos serviços, especialmente em um contexto de reorganização da Rede em fase de descensão da Pandemia. Isso exigiria uma atualização periódica do material construído, cabendo à gestão assumir essa responsabilidade.

A ideia é garantir o acesso a uma saúde de qualidade para todos. Para o Institute of Medicine dos Estados Unidos a boa qualidade em saúde, por sua vez, significa disponibilizar aos pacientes serviços adequados de uma forma tecnicamente competente, com boa comunicação, decisões compartilhadas e sensibilidade cultural. Nesse contexto, propõe-se como grandes objetivos a serem melhorados: a segurança, evitando lesões ao paciente; a eficiência, evitando o uso excessivo ou insuficiente dos serviços; a assistência centrada no paciente, garantindo que os valores do paciente orientem todas as decisões clínicas; a eficiência, evitando desperdícios; a equidade, disponibilizando serviços que não tenham variação em qualidade por causa de características pessoais; e as questões relativas ao acesso (Cobaito \& Cobaito, 2021).

Ademais, de modo a pensar melhor a organização de uma Rede local de Saúde, é válido identificar na literatura os principais parâmetros utilizados na avaliação da qualidade em saúde. Nesse contexto, o médico e pesquisador Avedis Donabedian destacou-se por ter estabelecido os sete pilares, atributos, dimensões ou domínios da qualidade em saúde. São eles: a eficácia, que tem relação com a capacidade da arte ou ciência da medicina produzir melhorias na saúde e no bem-estar; a efetividade, atrelada à melhoria na saúde, alcançada ou alcançável nas condições usuais da prática cotidiana; a eficiência, que corresponde à medida do custo com o qual uma dada melhoria na saúde é alcançada; a otimização, tornando-se relevante à medida que os efeitos do cuidado da saúde não são avaliados em forma absoluta, mas relativamente aos custos; a aceitabilidade, sinônimo de adaptação do cuidado aos desejos, expectativas e valores dos pacientes e de suas famílias; a legitimidade, relacionada a aceitabilidade do cuidado da forma em que é visto pela comunidade ou sociedade em geral; e a equidade, que diz respeito ao princípio pelo qual se determina o que é justo ou razoável na distribuição do cuidado e de seus benefícios entre os membros de uma população (Albuquerque et al., 2017).

A fim de avaliar a qualidade do acesso, a literatura também apresenta diversas categorias entre as quais são comuns a acessibilidade geográfica, disponibilidade, viabilidade e aceitabilidade. Em cada uma delas há um conjunto de barreiras, distribuído entre oferta e demanda por serviços de saúde. A acessibilidade geográfica diz respeito à adequação da localização dos serviços de saúde em relação à localização dos usuários, levando em conta distância, meios de transporte e tempo de deslocamento. A disponibilidade reflete a existência dos serviços e recursos de saúde em quantidade e qualidade apropriadas às necessidades dos usuários e a maneira pela qual os recursos são organizados para atender aos usuários. A viabilidade é o grau de adequação entre o custo da utilização dos serviços de saúde e a capacidade de pagamento dos indivíduos. A aceitabilidade diz respeito a percepções e atitudes dos usuários em relação a profissionais e práticas, e à percepção e atitudes dos profissionais e provedores a respeito das características pessoais dos usuários. De acordo com a Joint Commission on Accreditation of Healthcare Organization, acesso diz respeito ao grau com que o cuidado e a intervenção adequados estão disponíveis para 
responder às necessidades dos pacientes. Essa definição também é assumida pelo Observatório Europeu de Políticas e Sistema de Saúde, cujos integrantes defendem que o acesso a serviços de saúde constitui precondição de cidadania, sendo consensual que sua distribuição obedeça às necessidades de saúde. Esse conceito reconhece a necessidade de interação entre sistemas de saúde e indivíduos, famílias e comunidades onde vivem. Para avaliar a qualidade do acesso, a literatura apresenta diversas categorias entre as quais são comuns a acessibilidade geográfica, disponibilidade, viabilidade e aceitabilidade (Oliveira et al., 2019).

$\mathrm{Na}$ fase de validação do instrumento, os formulários foram estruturados didaticamente em três etapas: a primeira delas voltada para a identificação dos juízes envolvidos nesse processo; a segunda etapa focada no processo de validação propriamente dita e a terceira destinada a sugestões ou comentários em relação ao Guia.

$\mathrm{Na}$ etapa de identificação, os juízes foram caracterizados de modo a garantir que a amostra intencional tenha sido escolhida baseada em critérios objetivos. Em relação aos Gestores, por exemplo, apenas quatro entre os cinco convidados colocaram-se à disposição para participar da etapa de validação, foram eles: o Coordenador de Controle e Avaliação do Município, a Coordenadora da Atenção Básica e dois Diretores Administrativos de duas diferentes unidades hospitalares. O seja, todos os entrevistados são pessoas que assumem funções importantes na gestão municipal por mais de 03 anos, além de se mostraram solícitos em contribuir com a construção do Guia. Já entre os profissionais de saúde que atuam na rede, todos os cinco aceitaram participar dessa etapa do estudo. Todos, por sua vez, trabalham em pelo menos duas unidades de atendimento em Pinheiro-MA e por mais de 03 anos.

Ainda nesse contexto de identificação dos envolvidos, a escolha por universitários no último ano do curso de Medicina e de Enfermagem na Universidade Federal do Maranhão campus Pinheiro baseou-se no entendimento de que esse público por quatro anos ou mais esteve inserido na rede de saúde do Município através dos campos de práticas frequentados ao logo da formação acadêmica. Acredita-se, com isso, que sejam capazes de contribuir com a construção desse instrumento, bem como opinar em torno do mapeamento da Rede SUS em Pinheiro-MA. Como descrito no quadro 3, foram entrevistados três acadêmicos de Medicina e os dois acadêmicos de Enfermagem e esses, por suas vezes, frequentaram seis ou mais unidades de atendimento disponíveis no Município, o que sugere certo entendimento no que diz respeito à Rede de Saúde local.

Além dos públicos-alvo já mencionados, estiveram também envolvidos nesse processo de validação cinco representantes dos usuários, sendo estes considerados os verdadeiros protagonistas e os principais beneficiados com a construção de um instrumento que visa mapear, direcionar e orientar sobre todos os serviços disponíveis. Para que isso fosse possível, buscou-se a participação de cidadãos comuns e lideranças civis dispostas a contribuir com essa etapa do trabalho. Todos os entrevistados residem no município por mais de cinco anos e frequentaram pelo menos duas unidades de atendimento ao longo do ano de 2020 e 2021.

Pelo que se observa na literatura, parece não haver um consenso sobre a adequada seleção dos juízes-especialistas, de forma que se faz necessário escolher um sistema de classificação/pontuação que demonstre a expertise deles, tendo em vista sua qualificação e experiência, assim como sua aderência com a área de interesse do estudo. Nesse sentido, é importante buscar a participação de um número de avaliadores que viabilize a análise do material conforme a metodologia proposta. Da mesma forma, a heterogeneidade do grupo de especialistas é aconselhada para assegurar a validade dos resultados, já que a multidisciplinaridade permite obter consensos preditivos mais válidos. Além disso, tem-se mostrado oportuno aos avaliadores, quer sejam experts ou representantes do público-alvo, a possibilidade de escrever livremente suas opiniões, sem ater-se somente a responder numericamente o solicitado no instrumento de avaliação da tecnologia. Isso porque as anotações apresentadas, para além do que consta no instrumento avaliativo, podem ser úteis para obtenção de críticas e sugestões, assim como para o esclarecimento de pontuações atribuídas no instrumento, além de facilitar a compreensão da perspectiva do avaliador (Campos et al., 2021). 
Finalizada a etapa de identificação e caracterização dos juízes, partiu-se para a fase de interpretação da validação propriamente dita. Para isso, foi realizado o cálculo do Índice de Validade de Conteúdo (IVC) conforme descrito na metodologia deste trabalho. Sendo assim, em relação ao item "Linguagem" o ICV foi de 89,47\%, ou seja, dos 19 entrevistados 17 consideraram "muito clara" a linguagem empregada no Guia, sendo essa a resposta considerada satisfatória. Apenas 10,52\% marcaram a opção "Parcialmente clara" e $0 \%$ a opção "Confusa". Significa, portanto, que a maioria dos participantes acredita que o material supre as necessidades de compreensão a partir do emprego de uma linguagem clara e objetiva. No que diz respeito ao item "Acessibilidade", por sua vez, o ICV de 100\% comprova que os juízes consideraram o Guia uma ferramenta que proporciona uma facilidade na aquisição de informações, já que todos os envolvidos marcaram a opção "fácil acesso às informações" ao item em questão. Por outro lado, no que se refere à "Organização", 15,79\% marcaram a opção "parcialmente organizado" e 84,21\% a opção "bem organizado", sendo esse último o ICV correspondente ao item. Dessa forma, acredita-se que para a maioria dos entrevistados o Guia dispõe de uma estrutura ordenada, o que facilita o direcionamento na busca por informações. Quanto à "Relevância", todos acreditam se tratar de um material de "muita relevância", ICV de 100\%, além de terem respondido "sim" quando questionados sobre a vontade de ter acesso ou não ao Guia. A interpretação desses itens permite inferir, pois, que Guia da Rede SUS em Pinheiro-MA se trata de um instrumento de grande importância para todos, independente do papel social que desempenham a nível municipal. Por fim, dos 19 juízes envolvidos 09 deixaram sugestões ao final do formulário, sendo grande parte acatada pelo autor na elaboração da versão final do Guia.

Figura 2. Itens de validação do Guia da Rede SUS em Pinheiro-MA.

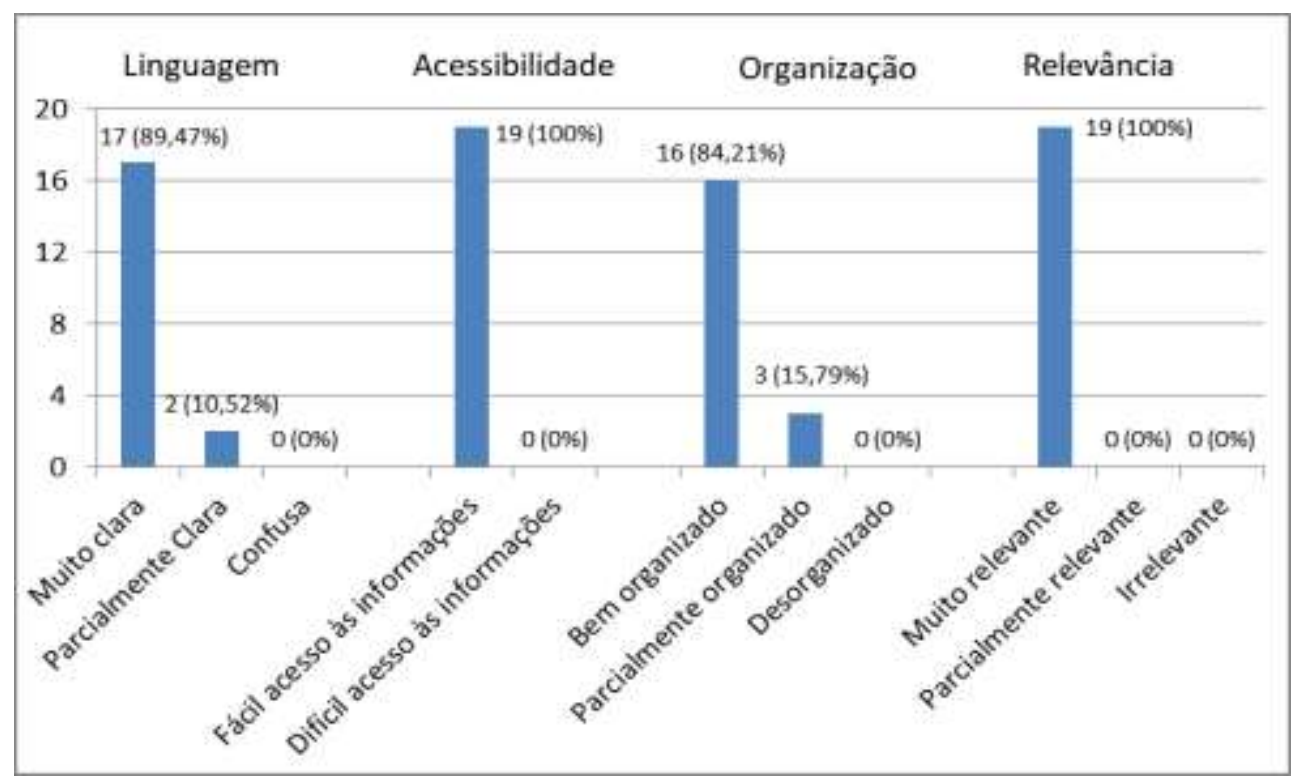

Fonte: Autores (2021).

Como não há teste estatístico para avaliar especificamente a validade do conteúdo, geralmente os pesquisadores usam uma abordagem qualitativa, através de um comitê de especialistas, e, em seguida, uma abordagem quantitativa, descritiva, usando o Índice de Validade de Conteúdo. Contudo, a validação apesar de ser uma etapa fundamental no processo de desenvolvimento e adaptação de instrumento de medidas, apresenta limitações por ser um processo subjetivo. Sendo assim, são necessárias aplicação de outras medidas psicométricas adicionais (Abreu et al., 2021).

Para avaliação da concordância do instrumento como um todo foi realizado o cálculo do Coeficiente de Validade de Conteúdo (CVC) a partir da soma dos Índices de Validade de Conteúdo (IVC) dividido pelo total de itens. Esse cálculo 
resultou em uma porcentagem de concordância de $93,42 \%$, atendendo, portanto, o valor mínimo $\geq 80 \%$. Diante dessa análise é possível comprovar a validade do instrumento, que, portanto, atende ao propósito para o qual foi elaborado.

Além disso, ainda nessa etapa de validação, evidenciou-se uma rica contribuição em experiências e conhecimentos, por parte dos juízes selecionados, no que diz respeito ao funcionamento da Rede SUS em Pinheiro-MA. Isso porque, ainda que o estudo tenha atendido ao propósito para o qual foi elaborado, alguns questionamentos e sugestões foram levadas em consideração na elaboração da versão final do instrumento. Pretende-se, com isso, estimular a existência um sistema de saúde em rede, que supere o isolamento dos serviços em níveis de atenção, o que produz baixa comunicação entre as equipes e consequente fragmentação do cuidado, além de dificuldades de continuidade da ação clínica pela equipe que cuida do usuário. Almeja-se também fomentar processos de co-gestão, valorizando e incentivando a inclusão dos trabalhadores e usuários em todo processo de produção da saúde (Faria, 2020), além de otimizar o funcionamento das organizações de forma a obter o máximo de eficiência (relação entre produtos e recursos empregados), eficácia (atingimento dos objetivos estabelecidos) e efetividade (resolução dos problemas identificados) (Silva et al., 2015).

Apesar de ser conhecimento inerente à psicologia, o modelo de Pasquali, tem sido observado em pesquisas de enfermagem para elaboração e validação de instrumentos em três fases de procedimentos: teóricos, empíricos e analíticos. Segundo Pasquali, os procedimentos teóricos contemplam a fundamentação teórica sobre o construto para o qual se pretende construir um instrumento de medida, aqui serão estabelecidas as definições das propriedades do construto, a dimensionalidade dos atributos, elaboração dos itens e validação de conteúdo. Nos procedimentos empíricos, serão definidas as técnicas de aplicação do instrumento piloto, para avaliar as propriedades psicométricas do instrumento. Já nos procedimentos analíticos, como no caso do trabalho em questão, são realizadas análises estatísticas para determinar a validade do instrumento (Medeiros et al., 2015).

Em suma, após incessante busca e leitura na literatura científica, observou-se um escasso número de estudos relacionados à construção e validação de instrumentos, ainda mais no formato de Guia e no contexto da Rede de Saúde local. O que se nota é que o produto desenvolvido neste estudo se trata de uma novidade ao contemplar, apesar de suas limitações, um exemplar inédito do que poderá repercutir positivamente na organização da Saúde Pública local de outros municípios e no acesso mais democrático às informações.

\section{Considerações Finais}

Instrumentos editoriais, como é o caso do Guia da Rede SUS em Pinheiro-MA, são conteúdos que têm por finalidade reconhecer, determinar, normalizar, padronizar ou regular ações e procedimentos. No caso em questão, levando em consideração a realidade da saúde local de um importante município localizado na microrregião da Baixada Maranhense, pretende-se garantir que usuários, profissionais da rede, gestores e acadêmicos da área da saúde reconheçam a Rede onde estão inseridos, seja com a finalidade de facilitar o acesso, integrar os setores, identificar áreas descobertas, bem como estimular a intervenção em níveis de atenção ainda pouco assistidos.

A expectativa é que este trabalho inovador e estruturante apoie todos os envolvidos, direta ou indiretamente, contribuindo com o processo de organização e planejamento da Rede, em especial daqueles que, no cotidiano do seu trabalho, têm a responsabilidade de viabilizar a implementação local de uma assistência à saúde contínua, articulada, integrada e solidária, reunindo condições singulares para que o SUS exercite, em plenitude, os seus princípios, além de contribuir com o que constitui o seu propósito basilar que é a qualidade de vida das pessoas. Assim sendo, pretende-se com este trabalho fortalecer a cidadania e garantir o exercício do direito à saúde, facilitando o acesso de todos os cidadãos aos equipamentos da rede pública de saúde, além de existir espaço também para atualizações futuras em relação à versão atualmente construída. 
Em resumo, conclui-se que o instrumento criado é apropriado para o alcance dos seus objetivos, já que os especialistas consideraram o Guia adequado quanto aos itens referentes à linguagem, acessibilidade, organização e relevância. Trata-se, pois, de uma tecnologia passível de ser reproduzida em qualquer localidade, servindo de inspiração e contribuindo para organização do processo de trabalho em todos os níveis de atenção à saúde.

Este trabalho se torna ainda mais relevante por permitir que outros tantos sejam desenvolvidos posteriormente. É necessário, por exemplo, avaliar a curto, médio e longo prazo a repercussão desse material após sua circulação na comunidade local ou, ainda, fazer um comparativo do perfil da Rede de Saúde do Município em momentos diferentes, permitindo traçar os principais avanços e dificuldades enfrentados ao longo do tempo. É possível também criar inclusive uma "Plataforma online" que permita realizar, por parte da gestão municipal e de maneira mais instantânea, alterações dos dados contidos no Guia, de modo a proporcionar atualizações mais instantâneas e garantir informações mais coerentes com a realidade. Ademais, é ainda oportuno se pensar em futuros estudos metodológicos de validação de Guias utilizados para organizar rede de saúde, a fim de obter melhorias e adequações ao que se propõe o Sistema Único de Saúde em seus princípios.

\section{Referências}

Abreu, R., Carioca, A., Sampaio, H., \& Vasconcelos, C. (2021). Validação de Instrumento de Avaliação de Materiais Educativos Impressos com foco no Letramento em Saúde para o Brasil 9AMMELS-BR). Research, Society and Development, 3.

Albuquerque, C., Madeira, G., Capanema, I., \& Ferreira, J. (2017). Qualidade em Saúde: evolução e desafios no contexto brasileiro. Buenos Aires: Business Assurance Usiness.

Albuquerque, M., \& Viana, A. (2015). Perspectivas de região e redes nas política de saúde brasileira. Saúde Debate, 29.

Alves. (2017). A dicotomia do Puncípio da Integralidade do SUS. Cadernos Ibero-Americanos de Direito Sanitário, 160.

Campos, D., Silva, L., Reis, A., Góes, F., Moraes, J., \& Aguiar, R. (2021). Elaboração e validação de vídeo educativo para prevenção de queda em criança hospitalizada. Texto \& Contexto Enfermagem, 12-13.

Cobaito, F., \& Cobaito, V. (2021). SUS-Sistema Único de Saúde: a gênese, contemporaneidade, e os desafios do amanhã. Inova Saúde, 161-162.

Cruz, A., \& Medeiros, A. (2021). Construção teórico-metodológicas de uma pesquisa: uma análise do caminho percorrido. Research, Society and Development, 5 .

Drumond, A., \& Neto, D. (2021). Gestão do SUS na perspectiva da literatura: fragilidades, potencialidades e propostas. Espaço para a Saúde, 3.

Faria, R. (2020). A territorialização da Atenção Básica à Saúde do Sistema Único de Saúde do Brasil. Ciência \& Saúde Coletiva, 4523.

Feliciello, D. (2021). Aspectos conceituais e operacionais do planejamento em saúde com ênfase no SUS. Campinas: Caderno de Pesquisa NEPP.

Gil, C., Luiz, I., \& Gil, M. (2016). O processo de Trabalho na Gestão do SUS. EDUFMA.

Guarnieri, S. P. (2021). A Participação Popular na Saúde: Desafios e Potencialidades no Contexto Municipal. Revista Saúde em Redes, 3.

Kripka, R., Scheller, M., \& Bonotto, D. (2015). Pesquisa Documental: considerações sobre conceitos e características na Pesquisa Qualitativa. $4^{o}$ Congresso Ibero-Americano em Investigação Qualitativa (p. 243). Aracajú: ResearchGate.

Luz, H., Carmo, W., Carmo, S., Carmo, M., Pantoja, C., Guimalhães, R., et al. (2020). Democratização da saúde: desafios da municipalização e descentralização administrativa relacionado a participação e controle social. Brazilian Journal of Development, 510.

Medeiros, C., \& Gerhardt, T. (2015). Avaliação da Rede de Atenção à Saúde de pequenos municípios na ótica das equipes gestoras. Saúde Debate, 161 .

Medeiros, R., Júnior, M., Pinto, D., Vitor, A., Santos, V., \& Barichello, E. (2015). Modelo de validação de conteúdo de Pasquali nas pesquisas em Enfermagem. Revista de Enfermagem Referência, 128.

Ministério da Saúde. (2019). Programa Academia da Saúde: Caderno Técnico de Apoio à Implantação e Implementação. Editora MS.

Reis, A., Sóter, A., Furtado, L., \& Pereira, S. (2017). Reflexões para a construção de uma regionalização viva. Ciência \& Saúde Coletiva, 1048.

Silva, A., Menezes, H., Silva, H., Fonseca, M., Junior, A., \& Silva, R. (2021). Validação de cartilha para uso correto de equipamento de proteção individual no contexto da COVID-19. Texto \& Contexto Enfermagem, 11.

Silva, R., Jorge, M., \& Júnior, A. (2015). Planejamento, gestão e avaliação nas práticas de saúde. EdUECE.

Sousa, T., \& Sousa, A. (2019). Políticas Públicas em saúde: um artigo de revisão sobre o Sistema único de Saúde no Brasil. Revista Científica Multidisciplinar 
Research, Society and Development, v. 11, n. 1, e43311125192, 2022

(CC BY 4.0) | ISSN 2525-3409 | DOI: http://dx.doi.org/10.33448/rsd-v11i1.25192

Núcleo do Conhecimento , 2.

Thomas, L., \& Fontana, R. (2020). Uso das Tecnologias de Informação e Comunicação como meio educacional na saúde: revisão integrativa. Research, Society and Development, 3 .

Feliciello, D. (2021). Aspectos conceituais e operacionais do planejamento em saúde com ênfase no SUS. Caderno de Pesquisa NEPP.

Abreu, R., Carioca, A., Sampaio, H., \& Vasconcelos, C. (2021). Validação de Instrumento de Avaliação de Materiais Educativos Impressos com foco no Letramento em Saúde para o Brasil 9AMMELS-BR). Research, Society and Development, 3. 